\title{
Pycnonuclear reactions and the physics of neutron star interiors
}

\author{
Dmitry Yakovlev*独 \\ Ioffe Physical Technical Institute \\ Politekhnicheskaya 26, 194021, St.-Petersburg, Russia \\ E-mail: yakeastro.ioffe.ru
}

\section{Kseniya Levenfish}

Ioffe Physical Technical Institute

Politekhnicheskaya 26, 194021, St.-Petersburg, Russia

E-mail: kseneastro.ioffe.ru

\begin{abstract}
Pycnonuclear fusion reactions in dense stellar matter are discussed in the context of deep crustal heating of neutron stars in X-ray transients. These transients are binary systems containing a neutron star and a low-mass companion. Neutron stars undergo periods of accretion and quiescence. The accreted matter can penetrate deeply into their crust and warm up the entire star due to nuclear transformations in the inner crust, particularly due to pycnonuclear reactions. Radiation from the warmed up surface is detectable during quiescent periods and carries information on the physical state of superdense matter in neutron star cores. The physics of heating in the accreted matter is outlined. The theory is compared with observations of thermal radiation from neutron stars in X-ray transients during quiescent periods. The constraints on the properties of superdense matter which result from this comparison are summarized.
\end{abstract}

8th Conference Quark Confinement and the Hadron Spectrum September 1-6 2008

Mainz, Germany

*Speaker.

${ }^{\dagger}$ A footnote may follow. 


\section{Pycnonuclear burning and deep crustal heating}

Nuclear fusion reactions in normal stars occur in thermonuclear regime - Coulomb barrier is penetrated owing to the thermal energy of colliding nuclei. In this presentation, we discuss another, pycnonuclear reaction regime, where Coulomb barrier is penetrated due to zero-point vibrations of reacting nuclei arranged in a lattice. The thermonuclear regime is realized in a rather low-density and warm plasma. The pycnonuclear burning operates at high densities and not too high temperatures and is almost temperature independent (occurs even at $T=0$ ). The formalism of nuclear reactions in the pycnonuclear regime, as well as in the intermediate thermo-pycnonuclear regimes, was developed by Salpeter and van Horn [1]; the problem has been elaborated later (see references in [2]). Pycnonuclear burning is negligibly weak in ordinary stars but intensifies at high densities $\rho$ in compact stars (neutron stars and white dwarfs). For instance, carbon undergoes rapid pycnonuclear burning at $\rho \gtrsim 10^{10} \mathrm{~g} \mathrm{~cm}^{-3}$.

We outline the effects of pycnonuclear reactions in neutron stars. The composition and the equation of state (EOS) of superdense matter in neutron star cores are still largely unknown (e.g., Ref. [3]). For instance, the inner core may contain nucleon (or nuclear/hyperon) matter, pion- or kaon condensates, or quark matter. Neither of these hypotheses can be accepted or rejected at present, and pycnonuclear reactions can be used to constrain them.

Pycnonuclear reactions can be important in accreting neutron stars. Consider the evolution of an accreted matter element in such a star. The heat released due to the infall of matter and thermonuclear reactions in the surface layers is thought to be mainly radiated away by photons from the neutron star surface. The ashes of thermonuclear burning sink then gradually into the star under the weight of newly accreted material and undergo further transformations [5, 6, 4] (beta captures; absorption and emission of neutrons; pycnonuclear reactions). The main energy release occurs [5, 6] in the inner crust, at densities from $\sim 10^{12}$ to $\sim 10^{13} \mathrm{~g} \mathrm{~cm}^{-3}$, about $1 \mathrm{~km}$ under the surface, in pycnonuclear reactions involving very neutron-rich nuclei (e.g., the reaction ${ }^{34} \mathrm{Ne}+{ }^{34} \mathrm{Ne} \rightarrow{ }^{68} \mathrm{Ca}$ at $\rho \approx 2 \times 10^{12} \mathrm{~g} \mathrm{~cm}^{-3}$ in the scenario of [5]). Specific nuclear transformations and the composition of the accreted crust depend on the composition of ashes but the total energy is robust, $\sim 1.5-1.9 \mathrm{MeV}$ per one accreted nucleon. The total energy generation rate, which produces deep crustal heating, is determined by the mass accretion rate $\dot{M}$ :

$$
L_{\mathrm{dh}} \approx(1.5-1.9) \mathrm{MeV} \dot{M} / m_{\mathrm{N}} \approx(0.9-1.1) \times 10^{34} \dot{M} /\left(10^{-10} M_{\odot} \mathrm{yr}^{-1}\right) \mathrm{erg} \mathrm{s}^{-1}
$$

( $m_{\mathrm{N}}$ is the nucleon mass, and $M_{\odot}$ is the solar mass). Thermal conduction spreads this heat over the entire star and warms the star.

\section{Theory versus observations of $X$-ray transients}

It is possible that the deep crustal heating manifests itself in X-ray transients which are binaries containing a neutron star and a low-mass companion [7]. They undergo the periods of outburst activity (days-months, even years) followed by the periods of quiescence (months-decades). Outburst activity is powered by accretion from an accretion disk. The accretion energy release at the neutron star surface makes a transient a bright X-ray source, with the luminosity $L_{X} \sim 10^{36}-10^{38}$ 
$\mathrm{erg} \mathrm{s}^{-1}$. The accretion is switched off or strongly suppressed during quiescent episodes when $L_{X}$ drops below $10^{34} \mathrm{erg} \mathrm{s}^{-1}$.

Although the nature of the quiescent emission is still uncertain, it can be powered [8] by the deep crustal heating. The heating during especially long outburst periods in some X-ray transients (e.g., KS 1731-260 and MXB 1659-29; see [10, 14-16] and references therein) overheats the crust. After the return to quiescence, the crust thermally relaxes to the stellar core, that is observed as a drop of the thermal X-ray luminosity to some constant level on timescales of $\sim 1 \mathrm{yr}$. In other transients the crust heating is not strong; their quiescent thermal emission is quasistationary reflecting that the crust is in the thermal equilibrium with the core.

Let us analyze these thermally equilibrated states. They are determined by the thermal balance between the time-averaged deep crustal heating and neutron star cooling. The averaging should be done over neutron star cooling time scales $(\sim 1-10 \mathrm{kyr})$. The time-averaged crustal heat power (1.1) is defined by the time-averaged mass accretion rate $\dot{M} \equiv\langle\dot{M}\rangle$. The crustal heat is partly radiated away by neutrinos from the entire star (mainly from its core), and the neutrino luminosity depends on the EOS in the core. The rest of the heat is carried by thermal conduction to the surface and radiated away by photons. It is also dependent of the core EOS, allowing one to constrain the EOS (see [9-12] and references therein).

We are mainly interested in the effects of the EOS on neutrino emission. Low-mass neutron stars possess the nucleon cores. Their neutrino emission is slow, being mostly determined by modified Urca and nucleon-nucleon bremsstrahlung processes. High-mass stars, in addition to the outer nucleon cores, possess the inner cores whose composition is unknown [3]. The neutrino emission from the inner core can be strongly enhanced by direct Urca process in nucleon (or nucleon-hyperon) matter or by somewhat weaker processes in pion-condensed matter, or by even weaker (but nevertheless intense) processes in kaon-condensed or quark matter.

Theoretically we can calculate heating curves $L_{s}^{\infty}(\dot{M})$, the dependence of the quasistationary photon thermal luminosity, redshifted for a distant observer, on $\dot{M}$. Fig. 1 presents such curves calculated with simplified models [12] for low-mass $\left(M=1.16 M_{\odot}\right)$ and high-mass $\left(M=2.04 M_{\odot}\right)$ neutron stars. The curves are quite common, and show no essential difference from those computed for more elaborated neutron star models [17].

Three panels correspond to different EOSs. The low-mass neutron star models are the same for the three cases; these stars are assumed to have a slow neutrino emission - neutron-neutron bremsstrahlung in the presence of strong proton superfluidity [12] which suppresses the modified Urca process. Three high-mass models are drastically different. The left panel is for the high-mass star with the fast neutrino emission due to the direct Urca process in the inner core. The middle panel refers to the high-mass star with the pion-condensed inner core, while the right panel to the high-mass star with the kaon-condensed (or quark) core; in both cases the direct Urca process is forbidden. A heating curve of a low-mass star gives an upper limit of $L_{s}^{\infty}$, whereas a heating curve of a high-mass star gives a lower limit of $L_{s}^{\infty}$. Varying the stellar mass from the lower values to the higher we obtain a family of heating curves which fill in the shaded space between the upper and lower curves.

Fig. 1 compares theoretical results with observations. The observational data are the same as in Ref. [17] (except for the source 4 whose data are taken from [14]). We treat $L_{s}^{\infty}$ as the quasi-stationary thermal surface luminosity of neutron stars in quiescent states, and $\dot{M}$ as the time- 

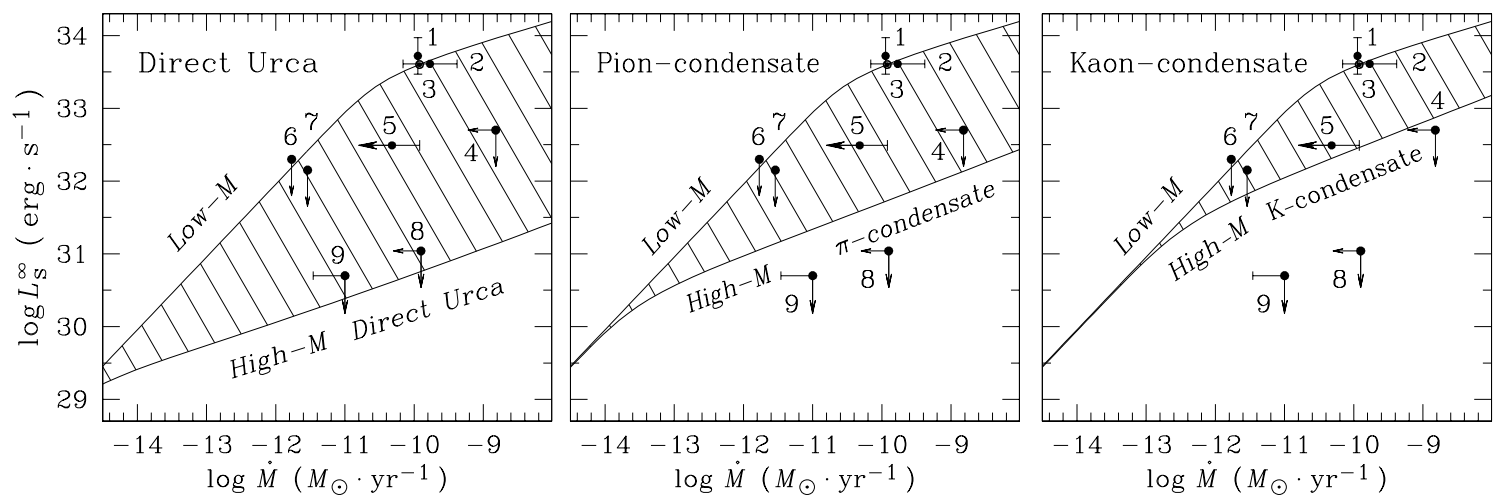

Figure 1: Allowable thermal states (shaded regions) of accreting neutron stars for three EOSs of their cores compared with observations of 9 X-ray transients: Aql X-1 (1), 4U 1608-522 (2), RX J1709-2639 (3); KS 1731-260 (4), Cen X-4 (5), SAX J1810.8-2609 (6), XTE J2123-058 (7), 1H 1905+000 (8), and SAX J1808.4-3658 (9).

averaged mass accretion rate (inferred from the fluence of X-ray emission in outburst states). The uncertainties of all data are large. Most of all, the theory deals with mass accretion rates averaged over periods of 1-10 kyr, while the observations cover the intervals of a few ten yr. Observations of outburst states are incomplete, and inferring $\dot{M}$ from these data is a complicated problem. Some mass accretion rates in Fig. 1 are upper limits. Some sources (first of all, 9 and 8) in quiescent state are very weak and show no thermal emission (with only upper limits on $L_{s}^{\infty}$ available).

Our present analysis is similar to that given in $[12,17]$. Although the data are uncertain, they confirm our previous conclusions [12, 17] (and similar conclusions of other authors; see, e.g., [913] and references therein). As seen from Fig. 1, 7 sources are consistent with all three model EOSs in neutron star cores. However, the two sources, 1H 1905+000 and SAX J1808.4-3658, seem to be too cold. They can be explained only by assuming that the neutron stars are massive and the neutrino emission from their cores is very strong. Its level should be as high as that provided by the direct Urca process in nucleon or nucleon/hyperon matter. The enhancement of the neutrino emission by pion or kaon condensates would be insufficient to explain such cold stars. In this respect, observations of SAX 1808.4-3658 are most crucial. If further observations confirm the present results, it would be a strong indication that direct Urca process is open in that neutron star.

Let us remark that the analysis of Fig. 1 allows one to test the same physics $[12,17]$ in the cores of accreting neutron stars in X-ray transients as the analysis of thermal radiation from cooling isolated neutron stars. Although the observational data on isolated neutron stars are currently more reliable, they are less restrictive (e.g., [18] and references therein) because of the absence of so cold isolated neutron stars as those in X-ray transients.

Thus, the pycnonuclear reactions in the deep neutron star crust can help solving the longstanding problem of the EOS of superdense matter in the neutron star core.

\section{Acknowledgments}

This work was supported by the grants of RFBR (08-02-00837) and FASI-RosNauka (NSh 2600.2008.2). 


\section{References}

[1] E.E. Salpeter and H.M. van Horn, Nuclear reaction rates at high densities, Astrophys. J. 155 (1969) 183.

[2] D.G. Yakovlev, L.R. Gasques, A.V. Afanasjev, M. Beard, and M. Wiescher, Fusion reactions in multicomponent dense matter, Phys. Rev. C 74 (2006) 035803 [astro-ph/ 0608488 ] .

[3] P. Haensel, A.Y. Potekhin, and D.G. Yakovlev, Neutron Stars. 1. Equation of State and Structure, Springer, New York, 2007.

[4] S. Gupta, E.F. Brown, H. Schatz, P. Möller, and K.-L. Kratz, Heating in the accreted neutron star ocean: implications for superburst ignition, Astrophys. J. 662 (2007) 1188 [ast ro-ph/ 0609828 ].

[5] P. Haensel and J.L. Zdunik, Non-equilibrium processes in the crust of an accreting neutron star, A\&A 227 (1990) 431.

[6] P. Haensel and J.L. Zdunik, Models of crustal heating in accreting neutron stars, A\&A 480 (2008) 459 [arXiv:0708.3996].

[7] W. Chen, C.R. Shrader and M. Livio, The properties of X-ray and optical light curves of X-ray novae, Astrophys. J. 491 (1997) 312 [astro-ph/9707138].

[8] E.F. Brown, L. Bildsten and R.E. Rutledge, Crustal heating and quiescent emission from transiently accreting neutron stars, Astrophys. J. Lett. 504 (1998) L95 [astro-ph/9807179].

[9] M. Colpi, U. Geppert, D. Page and A. Possenti, Charting the temperature of the hot neutron star in a soft X-ray transient, Astrophys. J. 548 (2001) L175 [astro-ph/ 0010572 ].

[10] R.E. Rutledge, L. Bildsten, E.F. Brown, G.G. Pavlov, V.E. Zavlin and G. Ushomirsky, Crustal emission and the quiescent spectrum of the neutron star in KS 1731-260, Astrophys. J. 580 (2002) 413 [astro-ph/0108125].

[11] E.F. Brown, L. Bildsten and P. Chang, Variability in the thermal emission from accreting neutron star transients, Astrophys. J. 574 (2002) 920 [astro-ph / 0204102 ].

[12] D.G. Yakovlev, K.P. Levenfi sh and P. Haensel, Thermal state of transiently accreting neutron stars, A\&A 407(2003) 265 [astro-ph/0209027].

[13] S. Campana, L. Stella, F. Gastaldello, S. Mereghetti, M. Colpi, G.L. Israel, L. Burderi, T. Di Salvo, and R.N. Robba, An XMM-Newton study of the $401 \mathrm{~Hz}$ accreting pulsar SAX J1808.4-3658 in quiescence, Astrophys. J. 575 (2002) L15 [astro-ph/ 0206376 ].

[14] E.M. Cackett, R. Wijnands, M. Linares, J.M. Miller, J. Homan, and W.H.G. Lewin, Cooling of the quasi-persistent neutron star X-ray transients KS 1731-260 and MXB 1659-29 MNRAS 372 (2006) 479 [astro-ph/0605490].

[15] P.S. Shternin, D.G. Yakovlev, P. Haensel, and A.Y. Potekhin, Neutron star cooling after deep crustal heating in the X-ray transient KS 1731-260, Mon. Not. Royal Astron. Soc. 382 (2007) L43 [arXiv:0708.0086].

[16] E.M. Cackett, R. Wijnands, J.M. Miller, E.F. Brown, and N. Degenaar, Cooling of the crust in the neutron star low-mass X-ray binary MXB 1659-29, Astroph. J. 687 (2008) L87 [arXiv:0806.1166].

[17] K.P. Levenfi sh and P. Haensel, Nucleon superfluidity versus thermal states of isolated and transiently accreting neutron stars, Astrophys. Space Sci. 308 (2007) 457 [astro-ph / 0611115 ].

[18] A.D. Kaminker, A.Y. Potekhin and D.G. Yakovlev, Cooling of isolated neutron stars as a probe of superdense matter physics, this volume. 